\title{
Magnetically tunable self-assembly of colloidal rings
}

\author{
Kwan H. Li and Benjamin B. Yellen ${ }^{\mathrm{a})}$ \\ Duke University, Department of Mechanical Engineering and Materials Science, Center for Biologically \\ Inspired Materials and Materials Systems, Durham, North Carolina 27708, USA
}

(Received 5 May 2010; accepted 5 August 2010; published online 25 August 2010)

\begin{abstract}
We present a technique using ferrofluid to induce bidisperse suspensions of superparamagnetic and diamagnetic beads to assemble into colloidal ring configurations. The separation distance between particles within the ring can be tuned by adjusting the ferrofluid concentration, which has the effect of enhancing the effective dipole moment of one of the components while screening the dipole moment of the other, leading to a wealth of different ring configurations. (C) 2010 American Institute of Physics. [doi:10.1063/1.3483137]
\end{abstract}

Ferrofluids (i.e., concentrated suspensions of $\sim 10 \mathrm{~nm}$ magnetic nanoparticles) are increasingly being used to direct the assembly of colloidal objects, including cells, ${ }^{1,2}$ microparticles, ${ }^{3-6}$ nanoparticles, ${ }^{7}$ nanowires, ${ }^{8,9}$ and liquid crystals, ${ }^{10}$ into arrangements which may have unique optical, electronic, and biological properties. In these examples, the ferrofluid serves as magnetic contrast medium, causing the nonmagnetic materials to respond to magnetic field gradients produced by other particles in the suspension as well as external field sources. Interactions between the different colloidal components can be tuned by adjusting the concentration of magnetic nanoparticles in the suspension to drive the particles to assemble into different structures. ${ }^{3,11}$ Most of prior investigations have restricted their attention to monocomponent colloidal suspensions to explore potential applications ranging from tissue engineering to the assembly of photonic crystals. More recently, the interactions within bicomponent and tricomponent suspensions have been investigated, where it was shown that a variety of geometric configurations, including, rings, poles, flowers, and two-tone structures, can spontaneously assemble in a uniform external magnetic field. ${ }^{3}$ Computational work by Engheta suggests that plasmonic nanoparticle rings are an ideal geometry for achieving negative refraction, ${ }^{12}$ in which the operating frequency can be tuned by adjusting the particle-particle separation distance within the ring. However, at present the lack of techniques for manufacturing colloidal nanorings in large quantities has impeded the development of these and other applications.

Here, we use a combination of theory and experiment to systematically study the magnetic field driven assembly of colloidal particle rings from bidisperse suspensions of superparamagnetic and diamagnetic microspheres. Although the scale of this self-assembly is at least an order of magnitude larger than the structures which can display negative optical refraction, these systems are more experimentally convenient since they allow for a direct statistical analysis of the assembly process using conventional optical microscopy and image processing techniques. Since the ferrofluid is much smaller than the colloidal objects of interest, it can be approximated as a magnetic continuum with magnetic permeability $\mu_{f}$. The spherical colloidal particles suspended inside ferrofluid are modeled as magnetic point dipoles, with an

${ }^{a)}$ Electronic mail: yellen@duke.edu. effective magnetic moment given by the following: ${ }^{13}$

$$
\vec{m}_{i}=3 \frac{\mu_{i}-\mu_{f}}{\mu_{i}+2 \mu_{f}} V_{i} \vec{H}_{i}=3 \bar{\mu}_{i} V_{i} \vec{H}_{i},
$$

where $V_{i}$ is the particle volume, $\vec{H}_{i}$ is the applied magnetic field at the particle center, and $\mu_{i}$ is the magnetic permeability of the $i$ th particle. A shorthand notation, $\bar{\mu}_{i}$, is used to denote the effective permeability for the $i$ th particle relative to the fluid. The potential interaction between two particles inside ferrofluid is equivalent to the interaction between two point dipoles as follows:

$$
U_{i j}=-\frac{\mu_{0}}{4 \pi}\left[\frac{3\left(\vec{m}_{i} \cdot \vec{r}_{i j}\right)\left(\vec{m}_{j} \cdot \vec{r}_{i j}\right)}{\left|\vec{r}_{i j}\right|^{5}}-\frac{\vec{m}_{i} \cdot \vec{m}_{j}}{\left|\vec{r}_{i j}\right|^{3}}\right],
$$

where the moments $\vec{m}_{i}$ of each particle are coupled together through the local fields and require a self-consistent solution. Our system consists of bidisperse colloids of radii, $r_{m}$ and $r_{n}$, with magnetic permeabilities, $\mu_{m}$ and $\mu_{n}$, where the subscripts $m$ and $n$ denote the superparamagnetic and diamagnetic components, respectively. We restrict our attention to ferrofluids with magnetic permeability satisfying the following condition: $\mu_{m}>\mu_{f}>\mu_{n}$, or in the shorthand notation $\bar{\mu}_{m}>0>\bar{\mu}_{n}$, in order to induce potential energy interactions between effectively positive and negative point dipoles. The self-assembly experiments were analyzed in a thin fluid film to create a quasi two-dimensional system, and the external field was applied in the normal direction (z-direction in Fig. 1) to induce repulsion between like components and attraction between unlike components. Adjusting the ferrofluid

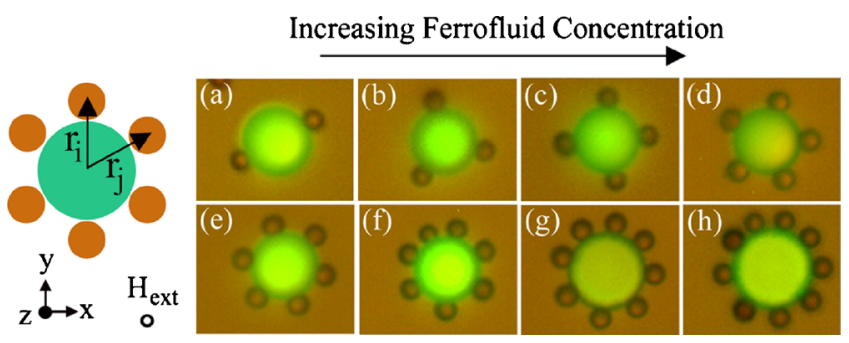

FIG. 1. (Color online) Magnetic assembly of $2.7 \mu \mathrm{m}$ superparamagnetic beads and $9.9 \mu \mathrm{m}$ diamagnetic green fluorescent beads is shown in an external field oriented along the normal (z) direction. (a)-(h) show the equilibrium structure as the ferrofluid volume fraction is increased from (a) $0.18 \%$, (b) $0.36 \%$, (c) $0.54 \%$, (d) $0.72 \%$, (e) $1.08 \%$, (f) $1.26 \%$, (g) $1.62 \%$, and (h) $1.8 \%$ where clearly the number of particles/ring grows with the ferrofluid concentration. 
permeability has the mutual effect of enhancing the dipole moments of one particle type while screening the moment of the other particle type. By tuning these attractive and repulsive interactions, it is possible to produce colloidal rings with controlled interparticle separation distances.

Equilibrium ring structures are determined by computing the ring configuration with the minimum potential energy as a function of the ferrofluid's magnetic permeability and the number of particles in the ring, $\mathrm{N}$. We consider a system in which one component type is large diamagnetic beads while the other type is smaller superparamagnetic beads. Due to the size difference, the diamagnetic bead serves as the core particle while the smaller superparamagnetic beads form the ring. The moments of each particle are computed selfconsistently through the following equation:

$$
\left[\begin{array}{l}
\vec{m}_{1} \\
\vec{m}_{2} \\
\cdots \\
\vec{m}_{N}
\end{array}\right]=\left[\begin{array}{cccc}
I & A_{12} & \cdots & A_{1 N} \\
A_{21} & I & \cdots & \cdots \\
\cdots & \cdots & \cdots & \cdots \\
A_{N 1} & \cdots & \cdots & I
\end{array}\right]^{-1}\left[\begin{array}{c}
3 \bar{\mu}_{1} V_{1} \vec{H}_{1}^{e x t} \\
3 \bar{\mu}_{2} V_{2} \vec{H}_{2}^{\text {ext }} \\
\cdots \\
3 \bar{\mu}_{N} V_{N} \vec{H}_{N}^{e x t}
\end{array}\right],
$$

where $\vec{H}_{i}^{\text {ext }}$ represents the external magnetic field at the $i$ th particle, $I$ is the identity matrix, and $A_{i j}$ is the interaction between the $i$ th and $j$ th particles in the ring. Once the moments are determined, the potential energy of the ring is computed by summation over all particles. For the sake of clarity, we break up the interaction energy into two parts as follows: $U=U_{\text {att }}+U_{\text {rep }}$ where $U_{\text {att }}$ represents the attractive interaction energy between the ring particles and the core, which for the geometry under consideration is given by following equation:

$$
U_{\mathrm{att}}=\sum_{i=1}^{N} \frac{\mu_{0} m_{n} m_{m}}{4 \pi\left(r_{n}+r_{m}\right)^{3}}
$$

while $U_{\text {rep }}$ represents the repulsive interaction between the particles in the ring. Due to repulsive interaction between ring particles, their equilibrium configuration should assume rotational symmetry to ensure maximum separation distance and lowest potential energy. The repulsive interaction can thus be represented by the following:

$$
U_{\text {rep }}=\sum_{i=1}^{N} \sum_{j \neq i}^{N} \frac{\mu_{0} m_{i} m_{j}}{8 \pi\left[\sqrt{r_{i}^{2}+r_{j}^{2}-2 r_{i} r_{j} \cos \left(\theta_{i j}\right)}\right]^{3}},
$$

where $m_{i}$ is the magnitude of the magnetic moment of the $i$ th ring particle, and $\theta_{i j}=2 \pi|i-j| / N$ is the angle between the $i$ th and $j$ th particles in the ring (see Fig. 1). The competition of repulsion interactions between the ring particles and attractive interactions between the ring/core will lead to a unique global energy minimum that can be tuned by adjusting the ferrofluid permeability. As the ferrofluid permeability is increased (leading to a commensurate increase in $\bar{\mu}_{n}$ and decrease in $\bar{\mu}_{m}$ ), the $U_{\text {att }}$ terms increase while the $U_{\text {rep }}$ terms decrease, leading to denser colloidal rings. Sparser rings form by decreasing ferrofluid concentration.

To test these predictions, we constructed an experimental system consisting of $2.7 \mu \mathrm{m}$ superparamagnetic beads (M270 Dynabeads, Invitrogen), $10 \mu \mathrm{m}$ diameter polystyrene green fluorescent beads (Duke Scientific), and EMG 707 ferrofluid (Ferrotec) that were mixed together in controlled ratios and observed through fluorescence microscopy. In all

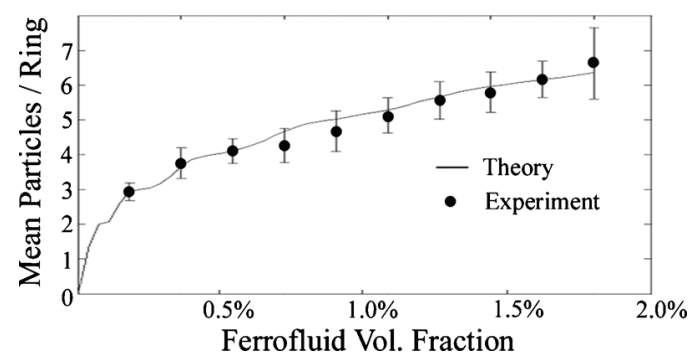

FIG. 2. The experimental data (points and standard deviation) shows the average number of ring particles around the core bead. The theoretically predicted ring/particle configuration (line) is also provided.

experiments, the concentrations of the superparamagnetic and diamagnetic beads were kept constant while the ferrofluid concentration was adjusted by systematically diluting it with water, maintaining a total volume of $300 \mu \mathrm{l}$ over all experiments. After vortexing, $15 \mu \mathrm{l}$ of the solution was placed between a glass slide and coverslip and sealed in with mineral oil to reduce evaporation and fluid motion. This fluid cell was then placed above a large solenoid. Its magnetic field was increased at a rate of $4 \mathrm{G} / \mathrm{min}$ over $15 \mathrm{~min}$, after which the field was maintained at $60 \mathrm{G}$. The slow field ramp rate provides time for the particles to assemble at quasithermodynamic equilibrium.

Obtaining high quality images was challenging due to optical attenuation of the ferrofluid at different concentrations. To increase the visibility of the particles, we applied masking tape to the bottom of the glass slide and conducted fluorescent reflectance microscopy, which permitted the nonfluorescent superparamagnetic beads to be observed through back scattering. Example micrographs of the assembly process are provided in Fig. 1.

Images of all the assembled structures within a $1 \mathrm{~cm}$ square region of the fluid were taken after $30 \mathrm{~min}$ to obtain the mean number of particles per ring and its variance. In order to avoid local depletion effects during the assembly process, we used an excess of the smaller superparamagnetic beads $\left(2 \times 10^{7}\right.$ particles $/ \mathrm{ml}$ and $2 \times 10^{9}$ particles $/ \mathrm{ml}$ for the diamagnetic and superparamagnetic beads, respectively, representing 100:1 mixing ratio between the particle number densities). Figure 2 depicts the experimental data of the average particle/ring number and its standard deviation as a function of the ferrofluid volume fraction. A theoretical fit is also provided which shows the expected particle/ring number obtained through minimization of the potential energy configuration using the magnetic permeability of the superparamagnetic bead and ferrofluid as the fitting parameters. The diameters of each bead were treated as random variables with a normal probability distribution as follows: $d_{n}$ $=9.9 \mu \mathrm{m} \pm 5 \%, d_{m}=2.7 \mu \mathrm{m} \pm 5 \%$, whereas the magnetic permeability of the superparamagnetic bead was assumed to follow a log normal distribution with $\mu_{m}=4 \mu_{0} \pm 15 \%$. A random number generator was used to determine values for the bead radii and permeability values over 1000 trials, and the equilibrium particle/ring structure was determined through minimization of the potential energy. The theoretically predicted mean particles/ring and standard deviation are provided using the best fits of $\mu_{m}=4 \mu_{0}$ and $\mu_{f}=\mu_{0}\left(1+\chi_{B} \phi\right)$, where $\chi_{B} \approx 21$ is the material susceptibility of bulk iron oxide and $\phi$ is the volume fraction of magnetic nanoparticles. 


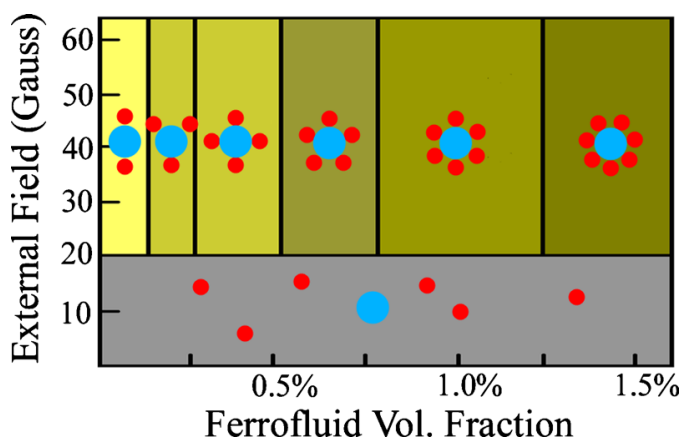

FIG. 3. (Color online) This phase diagram displays the regimes of stability for each colloidal ring structure as a function of the ferrofluid volume fraction. The structures begin to assemble at an average of $20 \mathrm{G}$; however this is a rough estimate and the transition may vary up to $10 \mathrm{G}$ in either direction.

We discovered that the best fit for the permeability of the superparamagnetic bead was several times larger than its moment measured in vacuum. ${ }^{14}$ One possible cause for this discrepancy is the Onsager theory for moments of molecules in liquids, ${ }^{15}$ in which he argued that a permanent dipole surrounded by a dense fluid of other dipoles will lead to a reaction field that enhances the effective polarizability of the dipole contained within the cavity of interest. This effect is in addition to the Clausius and Mosotti theory of Eq. (1). Since the superparamagnetic beads used in this work are known to have a slight permanent dipole, this effect can partially explain the larger fitting parameter. Another possible explanation is the breakdown of the continuum theory, in which concentration gradients in the ferrofluid ${ }^{7,16}$ (i.e., local ferrofluid aggregation around the superparamagnetic bead) can produce an effective dipole moment of the superparamagnetic bead that is larger than in vacuum. Using these reasonable fitting parameters, the rough trends are predicted quite well as verified by the strong agreement between theory and experiment (See Fig. 2) indicating the robust and tunable nature of the assembly technique. A phase diagram of the assembly process is provided in Fig. 3.

In conclusion, we present an inexpensive and simple magnetically driven self-assembly technique which uses ferrofluid to guide both positive and negative dipoles into a variety of ring structures. This magnetic assembly technique can potentially be scaled down into the submicron range. It is worth noting that a corollary assembly technique could be devised using optical or electric fields if suitable electric permeability differences between different particles and the fluid could be obtained. For example, the interaction between plastic spheres and gold nanoparticles in water could achieve a similar configuration by utilizing the surface plasmon resonance condition of gold to induce negative electric permeability when irradiated at optical wavelengths. Once assembled, these structures can be permanently bonded together either chemically or through molecular recognition elements, such as biotin and streptavidin or covalent crosslinking. Thus, we believe that this assembly process is quite general and future applications may exploit this process to construct nanorings in which the interparticle coupling can be tuned to create artificial structures of relevance to optical metamaterials and other fields.

The authors are thankful for support from the National Science Foundation Grant Nos. CMMI 0625480 and CMMI 0800173.

${ }^{1}$ M. D. Krebs, R. M. Erb, B. B. Yellen, B. Samanta, A. Bajaj, V. M. Rotello, and E. Alsberg, Nano Lett. 9, 1812 (2009).

${ }^{2}$ A. R. Kose, B. Fischer, L. Mao, and H. Koser, Proc. Natl. Acad. Sci. U.S.A. 106, 21478 (2009).

${ }^{3}$ R. M. Erb, H. S. Son, B. Samanta, V. M. Rotello, and B. B. Yellen, Nature (London) 457, 999 (2009).

${ }^{4}$ R. M. Erb, M. D. Krebs, E. Alsberg, B. Samanta, V. M. Rotello, and B. B. Yellen, Phys. Rev. E 80, 051402 (2009).

${ }^{5}$ A. T. Skjeltorp, Phys. Rev. Lett. 51, 2306 (1983)

${ }^{6}$ J. Černák, G. Helgesen, and A. T. Skjeltorp, Phys. Rev. E 70, 031504 (2004).

${ }^{7}$ R. M. Erb and B. B. Yellen, J. Appl. Phys. 103, 07A312 (2008).

${ }^{8}$ C. Ooi, R. M. Erb, and B. B. Yellen, J. Appl. Phys. 103, 07 E910 (2008).

${ }^{9}$ C. Ooi and B. B. Yellen, Langmuir 24, 8514 (2008).

${ }^{10}$ J. Rault, P. E. Cladis, and J. P. Burger, Phys. Lett. 32A, 199 (1970).

${ }^{11}$ B. B. Yellen, O. Hovorka, and G. Friedman, Proc. Natl. Acad. Sci. U.S.A. 102, 8860 (2005).

${ }^{12}$ A. Alu and N. Engheta, Phys. Rev. B 78, 085112 (2008).

${ }^{13}$ J. A. Stratton, Electromagnetic Theory (McGraw Hill, New York, 1941).

${ }^{14}$ B. B. Yellen, R. M. Erb, H. S. Son, R. Hewlin, Jr., H. Shang, and G. U. Lee, Lab Chip 7, 1681 (2007).

${ }^{15}$ L. Onsager, J. Am. Chem. Soc. 58, 1486 (1936).

${ }^{16}$ R. M. Erb, D. S. Sebba, A. A. Lazarides, and B. B. Yellen, J. Appl. Phys. 103, 063916 (2008). 